\title{
A Design of Wireless Sensor Network based on BLE
}

\author{
Guoliang Wei ${ }^{1, a}$ and Xiangbin $\mathrm{Ye}^{2, b}$ \\ ${ }^{1}$ Department of Instrument Science and Technology, National University of Defense Technology, \\ Changsha410073, China \\ ${ }^{2}$ Department of Instrument Science and Technology, National University of Defense Technology, \\ Changsha410073, China \\ alaoweiguoliang@qq.com, byxblyh@163.com
}

\begin{abstract}
Keywords: Wireless sensor network, Bluetooth Low Energy, Relay node
Abstract. With the release of super low-power consumption Bluetooth 4.0, the development of wearable intelligence devices is so fast in the world. In the field of wireless sensor net, to apply low-power consumption Bluetooth could decrease the cost of system and reduce the devices' volume, especially lower the power consumption. Based on Bluetooth Low Energy, this paper proposes a wireless sensor net integrate program and designs a key node in the wireless sensor net to reduce the node's volume and decrease the energy consumption. Through testing we found that the system could transfer the data which were collected by the sensors to the node by low-energy consumption Bluetooth. Due to its low cost and easy operation, the system is suitably applied to rigid monitoring systems such as military, industry, medical treatment, agriculture or life.
\end{abstract}

\section{Introduction}

Wireless sensor net is comprehensively applied on the field of military, industry, agriculture, smart house, environmental monitoring and medical treatment due to its huge advantage. By the advantage of super low energy consumption, Bluetooth 4.0 has been developing so fast after being applied in the wearable intelligible devices market. To study the wireless sensor based on the Bluetooth Low Energy has a profound effect on the application of wireless sensor net on more other fields, especially in the monitoring systems with more rigid standards in power consumption, cost and volume, and smart wearable devices.

\section{Bluetooth Low Energy}

Bluetooth Low Energy (BLE) is the lowest power consumption wireless technology for design and use, it is bluetooth 4.0. Classical Bluetooth which was designed to unite the global computer and communicating devices has been comprehensively applied in every sector. More and more applications ask for a wider band width of Bluetooth, so faster wireless electric technology is applied in the Bluetooth system. Bluetooth 3.0 has basically realized the physical data transfer speed up to hundreds of Mbps. But BLE chooses a completely different orientation: to optimize the system by lower the energy consumption as low as possible instead of increasing available data transfer speed. It's to say that may be we couldn't get a high transfer speed, but we can prolong the junction for more hours or even more days. Classical Bluetooth couldn't meet the demand of the devices supported by button battery for low energy, so the Bluetooth 4.0 is created.

BLE has been and will be widely used due to its low energy consumption in prior, and low cost and opening make it more available. The BLE communication protocol supports the communication of single-node (the host) to multi-nodes (subordinate engines). It can construct the net of BLE sensor nodes by design. So it could be applied on lower energy multi-node detecting system. BLE communication protocol will automatically reconnect the system when there is a break, so it could lower the energy consumption while simplify the process.

The BLE communication protocol is designed into layers. As showed Fig.1, the controlling layer includes physical layer, link layer and controlling entrance layer; the department of host engine 
includes logic link control and auto-adapting protocol layer, safeguard layer, property protocol layer, general accessing profile (GAP) layer and general attribute text (GATT) layer.

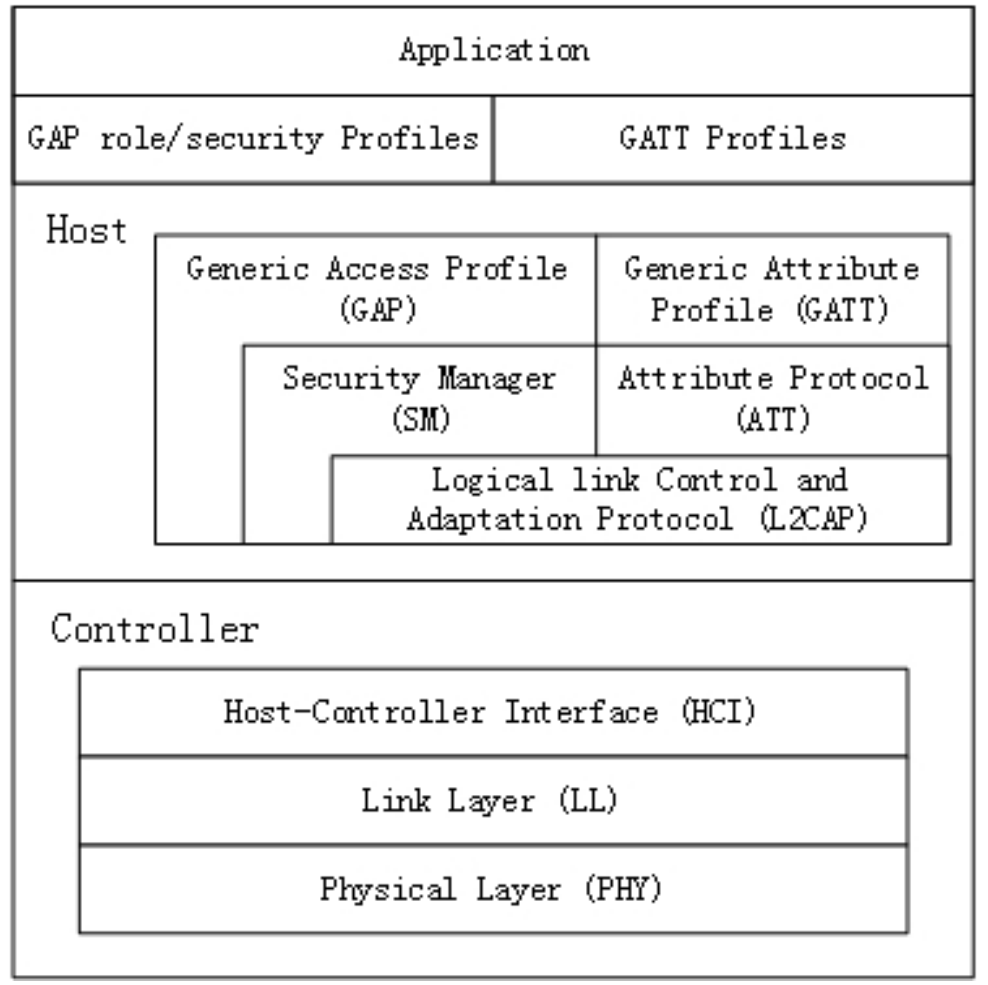

Fig. 1. The protocol of bluetooth low energy

The link layer and physical layer controls the working situation of the chip. After the sequential process of preparing, broadcasting, monitoring or scanning, launching and connected, the host engine will broadcast and accept the other engines as subordinate. The ATT layer defines the role of customer and servicer. Engines showing the property of data are the servicer and the matching ones are customers. The link layer's situation (the host and subordinate) and the process of defining role by ATT are mutual independent. The GATT layer defines the frame of ATT layer and stipulates the structure of allocation profile. All communication data of connected devices are processed by GATT's subroutine, and application programs and allocation profile applying the GATT layer directly. All layers mentioned above are supplied as source code.

The connecting model of BLE can be classified as host-subordinate model and broadcast-observer model. The host-subordinate model adopts star-shape topology structure which the host engine manage the connecting and multiple subordinate engines could be connected. The host- subordinate model is applied on the connecting nodes in this design.

\section{Wireless Sensor Net Integrate Design Based on BLE}

Intergrate Program Design. This paper adopts tree-shape topology structure to design the wireless sensor net, the structure is showed in Fig.2. The structure includes the sensor-nodes, relay nodes and root node (convergent node). Low energy consumption Bluetooth supports single-to-single or single-to-multiply connecting. Data transfer between nodes of the wireless sensor net is realized by adopting relay nodes in this paper.

The sensor-nodes catche the data by sensors and then transfer the data to relay nodes. The relay nodes read the data and transfer it to root node to make data summarized. The root node preprocesses the data and sends them to human-computer interaction terminal devices by available way such as WiFi or the Internet. 


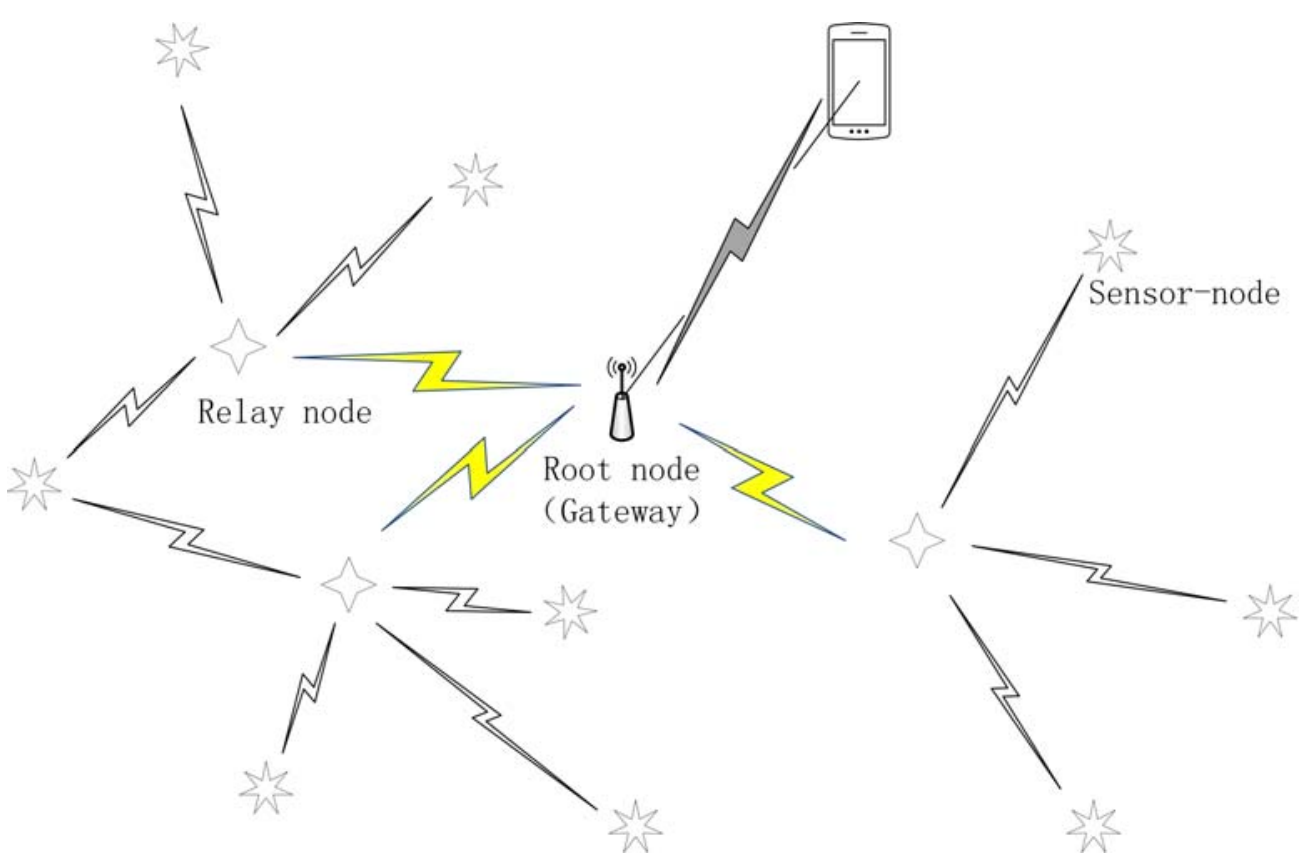

Fig.2 The construction of WSN based on BLE

The construct of the sensor-node and relay node are showed in Fig.3 and Fig.4. The host engine adopts CC2541 chip with core of the 51 single chip micro-computer, and running with BLE protocol stack, the chip can manage the BLE connecting and coordinate all the modules. Special applications should be developed to realize the function of root node on Android devices which accept BLE.

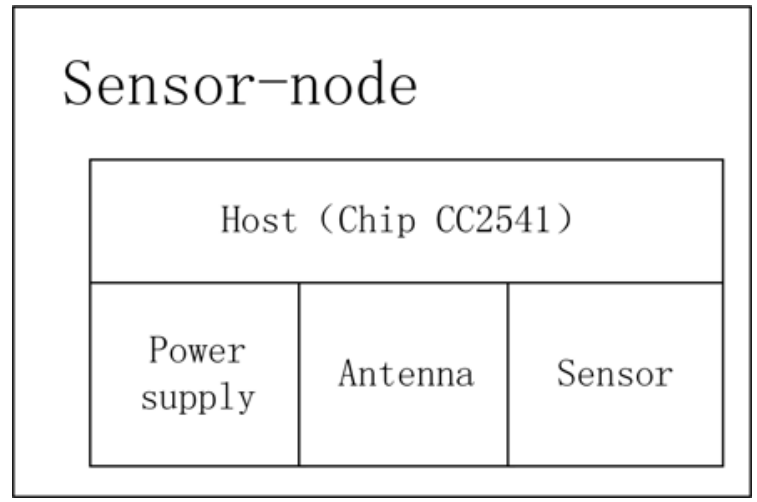

Fig. 3. The module of the sensor-node

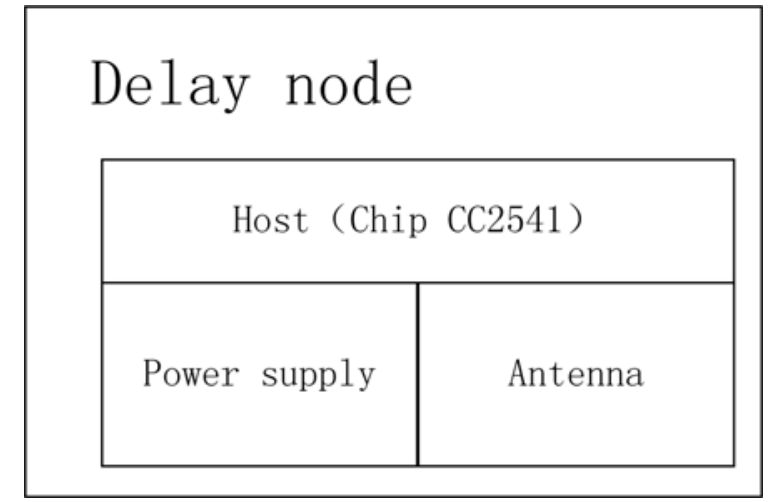

Fig.4. The module of delay node

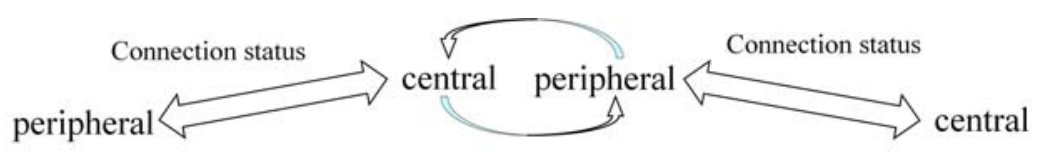

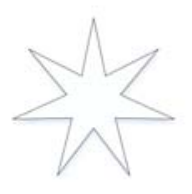

Sensor-node

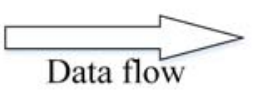

Data flow

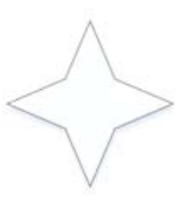

Relay node

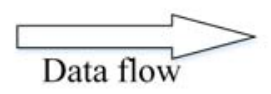

\section{Root node}

Fig.5. The process diagram of data transfer

The Process of Data Transfer. After the sensor catching the data, the broadcasting could be launched by BLE. Meanwhile, the relay node scans the device and find sensor node and then applies for connecting. Sensor node accepts the connecting application and makes itself the subordinate 
engine and makes the relay node the host engine. As the host engine, the relay node read the data transferred by the sensor node. When the setting time is up after successful connecting, the connecting will be break. And the sensor node will rebroadcast by BLE while waiting for reconnecting. Relay node and root node do the same connecting process while relay node works as subordinate engine and root node works as host engine. All the data will be collected to the root node. After finishing the data transfer, the system will scan devices and connect sensor node and read data again and again till the data transfer finished. During the process, the function roles which relay node plays is the key. Connecting process is showed in Fig. 5.

\section{Node Design}

Compare to sensor node design, the relay node design reduces the sensor module and adds a power management electric circuit. On account of repeating role transformation of relay node in the process while the nodes connected by BLE, the role transformation of relay node becomes the key.

Hardware Design. The core processor chip of the sensor node and the relay node in this design are adopted the chip CC2541 produced by TI company. The chip CC2541 includes the 51 processor and is compatible with Bluetooth protocol. It supports the Bluetooth communication and could process a certain data. It's significant to choose uniprocessor to reduce the system energy consumption and volume. And the entrance circuit of the chip of processor CC2541 is showed in Fig.6 .

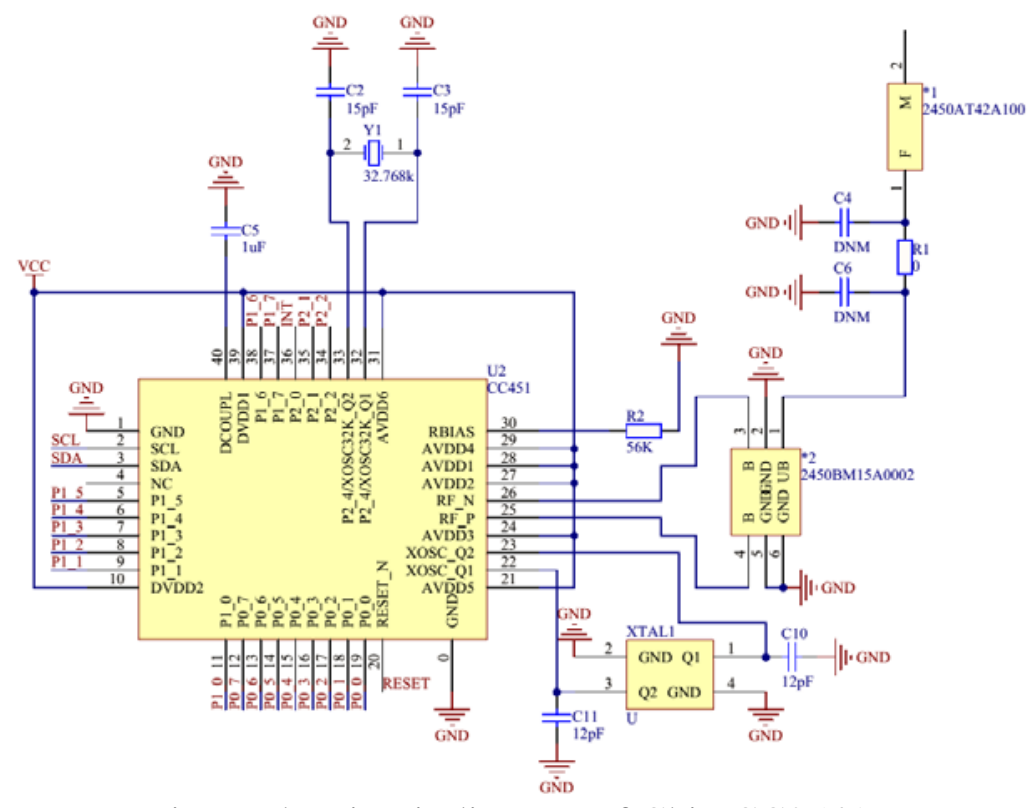

Fig.6. The circuit diagram of Chip CC2541

Node's communication module is composed of matching circuit and ceramic antennas. The front end of ratio frequency is adopted Balun match circuit. The structure of the circuit will determine the communication distance and system energy consumption directly. The design provided by TI company is reliable, so we design this part of the circuit according to it. The power adopts button battery which is expected to work for at least 6months continuously.

Software Design. Node's data collection, data processing, Bluetooth connecting and data transfer are all completed based on the BLE protocol. The BLE protocol provides a simply operation system which can satisfy the design. The relay node software design process is showed in Fig. 7.

During the transformation and connection of relay node, the software will control the system to restart and choose to enter the situation of scanning (connected with sensor node as host engine) or broadcasting (connected with root node as subordinate). During the process, to set reasonable node broadcasting interval, scanning interval and the switch frequency of relay node to ensure smooth data transfer. And adjust the connecting time and connecting interval of nodes according to different monitoring system. When the relay node is in the broadcasting situation, sensor nodes may be in the 
broadcasting situation as well. So the root node screens the scanned available devices and only connects the relay node.

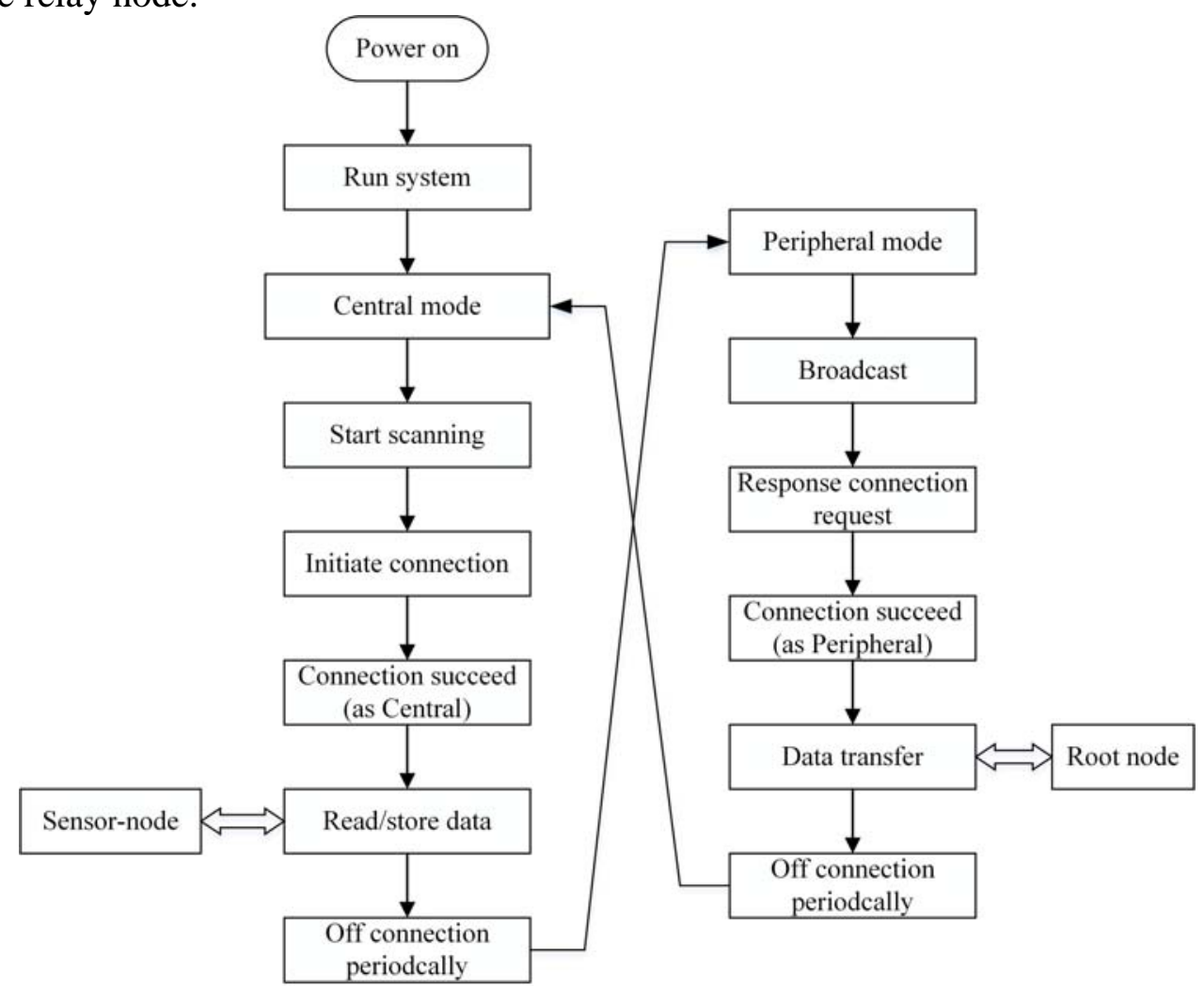

Fig.7. The software flow chart of the relay node

\section{Conclusion}

The nodes of wireless sensor work normally. The data collected by the sensor can get through the relay node normally and gather to the root node. We make a experiment than let the sensor-node sends two characters NU to relay node, and relay node sends them to root node(Android phone as an example), then the phone can get this two characters. The experiment effect is showed in figure 8 . The experiment proofs that the design is feasible. The design makes wireless sensor net which based on BLE come true.

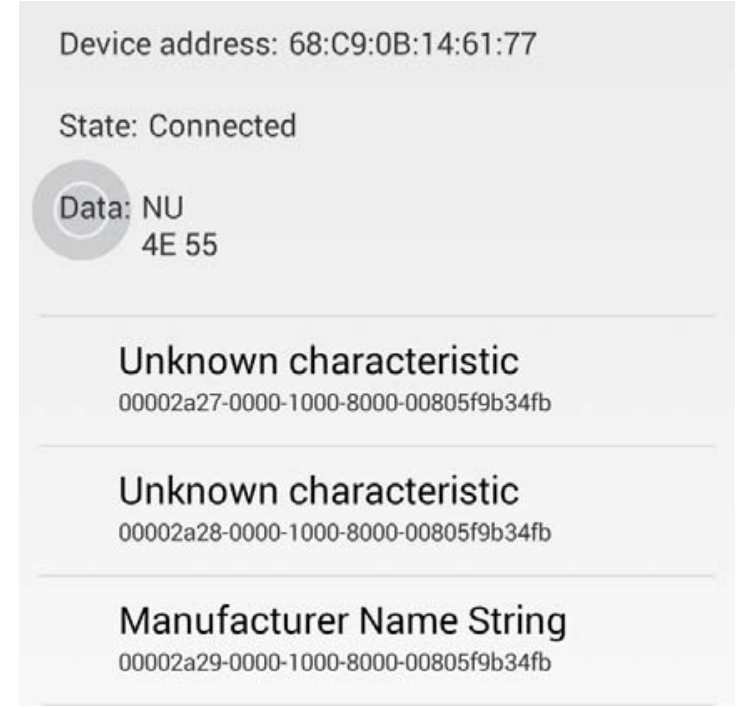

Fig.8. The experiment effect 


\section{References}

[1] Robin Heydon: Bluetooth Low Energy The Developer's Handbook (China Machine Press, China 2014).

[2] Xiaogang Guo, Siqi Feng: Fire Control Radar Technology Vol.45 No.1 p.89-91, p.100, In Chinese.

[3] Chun WU, Xuedong Chen, Hong Jiang, Hongyu Chu:Computer Applications and Software Vol.27 No.4 p.74-76, p.101, In Chinese. 